\title{
Pulmonary Valve Vena Contracta Area
}

National Cancer Institute

\section{Source}

National Cancer Institute. Pulmonary Valve Vena Contracta Area. NCI Thesaurus. Code C127591.

The area of the vena contracta of the pulmonic valve. 